\begin{tabular}{|c|c|}
\hline Title & A dvanced V alidation of Nonequilibrium Plasma Flow Simulation for A rc-Heated Wind Tunnels \\
\hline Author(s) & T akahashi, Y usuke; A be, T akashi; T akay anagi, Hiroki; Mizuno, Masahito; Kihara, Hisashi; A be, Ken-ichi \\
\hline Citation & $\begin{array}{l}\text { Journal of Thermophysics and Heat Transfer, 28(1), 9-17 } \\
\text { https://doi .org/10.2514/1.T } 3991\end{array}$ \\
\hline Issue Date & 201401 \\
\hline Doc URL & http://hdl.handle.net/2115/56573 \\
\hline Rights & (c) 2014 A merican Institute of A eronautics and A stronautics \\
\hline Type & article (author version) \\
\hline File Information & A dvanced V alidation of Nonequilibrium Plasma Flow Simulation for A rc-Heated Wind T unnels.pdf \\
\hline
\end{tabular}

Instructions for use 


\title{
Advanced Validation of Nonequilibrium Plasma Flow Simulation for Arc-Heated Wind Tunnels
}

\author{
Yusuke Takahashi ${ }^{1}$, Takashi $\mathrm{Abe}^{2}$, \\ Japan Aerospace Exploration Agency, 3-1-1 Yoshinodai Chuo-ku, Sagamihara, Kanagawa \\ 252-5210, Japan \\ Hiroki Takayanagi ${ }^{3}$, Masahito Mizuno ${ }^{4}$, \\ Japan Aerospace Exploration Agency, 7-44-1 Jindaiji-higashi-machi, Chofu, Tokyo \\ 182-8522, Japan \\ and \\ Hisashi Kihara ${ }^{5}$ and Ken-ichi Abe ${ }^{6}$ \\ Kyushu University, 744 Motooka Nishi-ku, Fukuoka 819-0395, Japan
}

\begin{abstract}
Turbulent plasma flows in arc heaters such as JAXA $750 \mathrm{~kW}$, NASA $20 \mathrm{MW}$, and Kyushu University $20 \mathrm{~kW}$ facilities were investigated, and the distributions of the flowfield properties were successfully obtained. The arc discharge in the constrictor section and the expansion processes in the nozzle section play key roles in the formation of an arc-heated flow. Hence, for accurately predicting high-enthalpy flow properties, it is important to model correctly the complex phenomena observed in various-scale facilities. For this purpose, an integrated analysis model to simulate various-scale arc-heated flows with high accuracy was developed. The turbulent flow field was described using the Reynolds-averaged Navier-Stokes equations with a multitemperature model, which was tightly coupled with electric-field and radiation-field calculations. A sophisticated and low-cost radiation model and a low-Reynolds number two-equation turbulence model were introduced into the flow-field simulation. To validate the present integrated analysis model, the computed results were compared with the corresponding experimental data for the mass-averaged enthalpy, translational and rotational temperatures, and number density of nitrogen obtained through spectroscopic and laser-induced fluorescence techniques. Moreover, the mechanisms of energy input by discharge and energy loss are discussed with the distributions of the electronic excitation temperature and heat flux on the constrictor wall derived from the arc column. Although the results indicate that a relatively detailed discharge model is required to describe the arc discharge with relatively high accuracy, the present flow-field model was generally in good agreement with various operating conditions of the facilities.
\end{abstract}

\section{Nomenclature}

$D=$ effective diffusion coefficient, $\mathrm{m}^{2} / \mathrm{sec}$

$e=$ electric charge, $\mathrm{C}$

$\mathbf{E}=$ Electric field vector, $\mathrm{V} / \mathrm{m}$

$\mathbf{F}=$ vector of numerical flux

\footnotetext{
${ }^{1}$ Aerospace Project Research Associate, Institute of Space and Astronautical Science (Currently: Hokkaido University, Kita13 Nishi8, Kitaku, Sapporo, Hokkaido, 060-8628, Japan; ytakahashi@eng.hokudai.ac.jp.)

${ }^{2}$ Professor, Institute of Space and Astronautical Science

${ }^{3}$ Researcher, Aerospace Research and Development Directorate

${ }^{4}$ Researcher, Aerospace Research and Development Directorate

${ }^{5}$ Assistant Professor, Department of Aeronautics and Astronautics

${ }^{6}$ Professor, Department of Aeronautics and Astronautics
} 


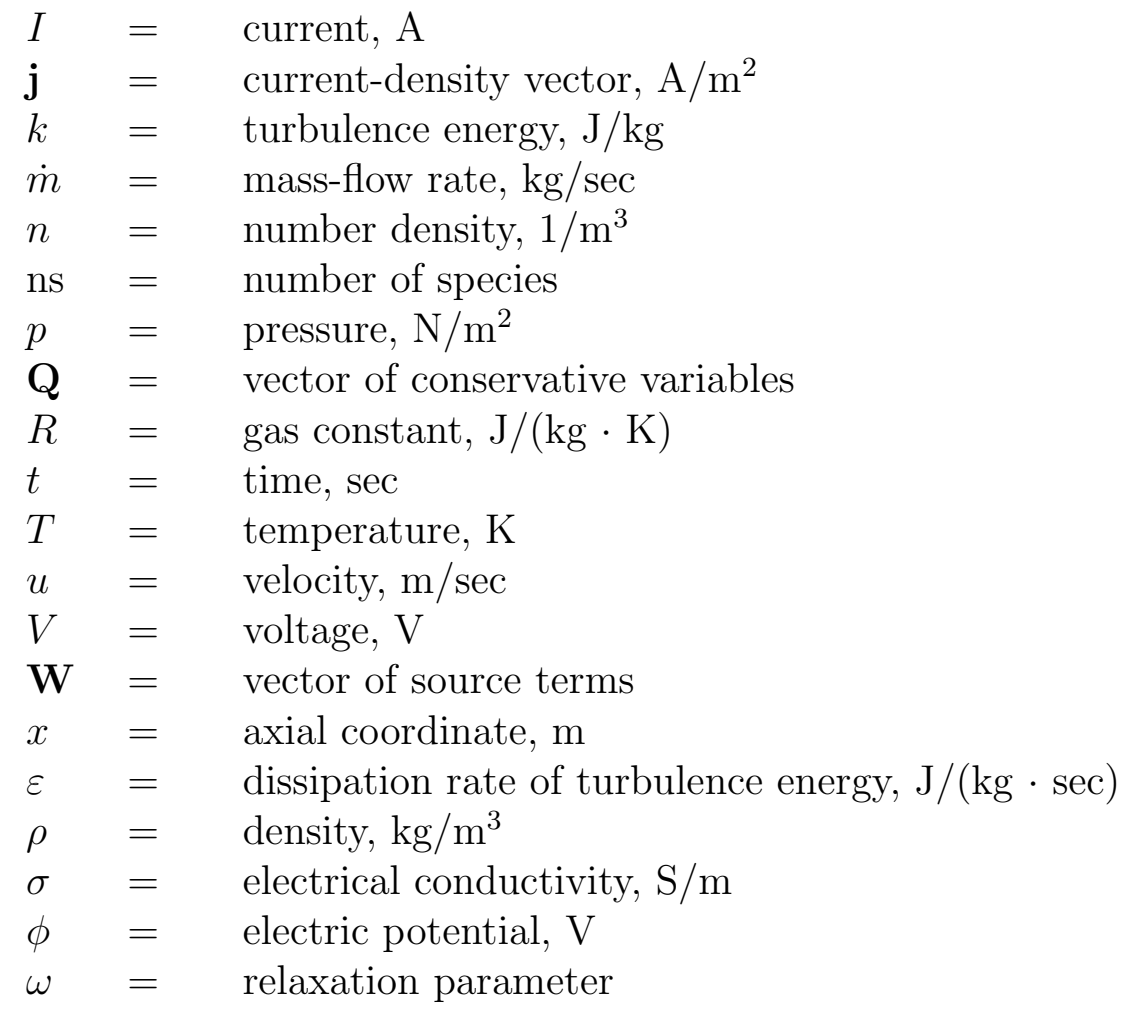

Subscripts

$\begin{array}{lll}\text { av } & = & \text { mass-averaged } \\ \mathrm{e} & = & \text { electron } \\ \mathrm{ex} & = & \text { electronic excitation } \\ \mathrm{rad} & = & \text { radiation } \\ \mathrm{rot} & = & \text { rotation } \\ s & = & \text { species } \\ \mathrm{t} & = & \text { turbulent } \\ \mathrm{tr} & = & \text { translation } \\ v & = & \text { viscous } \\ \mathrm{vib} & = & \text { vibration }\end{array}$

\section{Introduction}

An arc-heated wind tunnel is widely used for ground-based experiments of planetary entry environments. Its applications include performance tests of thermal protection systems. To perform such experiments with high accuracy, it is essential to understand the physical properties of the free jets exhausted from the nozzle. However, the flow field in an arc heater is complicated because of the complex phenomena associated with the heating process caused by the discharge in the constrictor as well as by the strong thermochemical nonequilibrium in the nozzle. Consequently, it is difficult to measure simultaneously all the properties of the free jets. Recently, because of the development of high-performance computers, numerical simulation has become a powerful tool for investigating the details of complex flow fields.

Several studies have investigated arc-heated flow properties such as enthalpy, temperature, velocity, and species concentration with numerical analysis methods [1-8]. To achieve 
accurate predictions with such simulations, it is critical to construct a detailed model of the heating process in the constrictor and the expansion phenomena in the nozzle section. For the heating process, it is preferable to simulate the flow field coupled with the electric-field calculation because the arc discharge strongly affects the formation of the arc-heated flow field. Moreover, because the expansion flow in the nozzle is excited rotationally and vibrationally, it is desirable to separate these temperatures from the translational temperature. It has been clarified in $[2,9]$ that the arc-heated flows in the Kyushu University wind tunnel (KUWT), which is a constrictor-type $20 \mathrm{~kW}$ arc heater, are in strong thermochemical nonequilibrium. In relatively large arc-heating facilities, e.g., the JAXA $750 \mathrm{~kW}$ segmented-type arc heater (JXWT) [10] and the NASA $20 \mathrm{MW}$ aerodynamic heating facility (AHF) [11], both turbulence and radiation are more important for heat transfer from the high-temperature gas (i.e., arc column) to the surrounding cold gas. Hence, it is important to model the radiation and turbulent heat transports in detail. However, thus far, few computational fluid dynamics (CFD) codes have been developed to simulate these arc-heated flows with an integrated analysis model. To increase the reliability of a physical model introduced into a CFD code, it is desirable for the model to be applicable to various types of arc heaters, including the constrictor-type (KUWT) and segmented-type (JXWT and AHF) facilities.

In our previous works $[12,13]$, in which we constructed an integrated model of the plasma flows in various arc-heated wind tunnels, we coupled a sophisticated radiation-model equation with the flow-field and electric-field equations. Further, a low-Reynolds-number two-equation turbulence model ( $k-\varepsilon$ model) was introduced into the simulation code for representing the turbulent phenomena. For expressing the thermal nonequilibrium in more detail, the present flow field is described by the Navier-Stokes equations with a multitemperature model. However, validation of the model is insufficient, although several comparisons with experimental data have been attempted.

Thus far, the Pitot pressure, heat flux, and mass-averaged enthalpy of the arc-heated flows have been measured though the efforts of many research groups. These experimental data have played major roles in validating CFD models, although they are inadequate for investigating discharge in the heating section and nonequilibrium in the expansion section. Recently, for the free jet in the vacuum chamber, spectroscopic analysis of the molecular band emission [14] and laser-induced fluorescence (LIF) [15-17] has been conducted for various archeated wind tunnels. Moreover, for the constrictor, the emission spectra of the arc column and the heat flux have been measured $[18,19]$. These efforts have revealed the distributions of translational temperature, flow velocity, and number density. In the present study, the objective is to validate the present integration analysis model to accurately predict the flowfield properties in various types of arc heaters and to clarify the information required to construct the next-generation flow simulation model with the newly measured data.

\section{Formulation}

\subsection{Governing Equations}

The physical models used here are basically the same as those used in our previous research on arc-heated flows $[12,13]$. In this section, the present governing equations and models are briefly described.

In this study, the following assumptions are employed. I) The flow is turbulent, steady, continuous, and axisymmetric. II) The test gas is nitrogen or air. III) The flow field is in 
thermochemical nonequilibrium, and the temperature is separated into translational $\left(T_{\mathrm{tr}}\right)$, rotational $\left(T_{\text {rot }}\right)$, vibrational $\left(T_{\text {vib }}\right)$, and electron $\left(T_{\mathrm{e}}\right)$ temperatures. Note that the electronicexcitation energy mode and the electron energy are assumed to be equilibrated (i.e., the electronic excitation temperature $T_{\mathrm{ex}}$ is equal to $T_{\mathrm{e}}$ ). IV) The Lorentz force, Hall current, and ion slip can be neglected because the arc current is too small to induce an effective magnetic field. V) Radiation is considered.

The flow field is described using the Reynolds-averaged Navier-Stokes (RANS) equations with a multitemperature model and an equation of state. The RANS equations comprise the conservations of total mass, momentum, total energy, species mass, rotation energy, vibration energy, and electron energy, and two transport equations of turbulent energy and its dissipation rate. The equation system can be written in a vector form as follows:

$$
\frac{\partial \mathbf{Q}}{\partial t}+\frac{\partial \mathbf{F}}{\partial x_{j}}=\frac{\partial \mathbf{F}_{v}}{\partial x_{j}}+\frac{\partial \mathbf{F}_{\mathrm{rad}}}{\partial x_{j}}+\mathbf{W} .
$$

The conservative vector is expressed as $\mathbf{Q}=\left[\rho, \rho u_{i}, E, \rho_{s}, E_{\mathrm{rot}}, E_{\mathrm{vib}}, E_{\mathrm{e}}, \rho k, \rho \varepsilon\right]$. Further, $\mathbf{F}, \mathbf{F}_{v}$, and $\mathbf{F}_{\text {rad }}$ represent the vectors of the inviscid, viscous, and radiation terms, respectively. Finally, $\mathbf{W}$ denotes the vector of the source term. The equation of state can be expressed as

$$
p=\sum_{s \neq \mathrm{e}}^{\mathrm{ns}-1} \rho_{s} R_{s} T_{\mathrm{tr}}+\rho_{\mathrm{e}} R_{\mathrm{e}} T_{\mathrm{e}}=\sum_{s \neq \mathrm{e}}^{\mathrm{ns}-1} \rho_{s} R_{s} T_{\mathrm{tr}}+p_{\mathrm{e}} .
$$

The governing equations are transformed into the generalized coordinate system and solved using a finite-volume approach. All the flow properties are set at the center of a control volume.

The transport properties such as viscosity, thermal conductivity, and binary diffusion coefficients for a mixture gas are evaluated using Yos' formula, which is based on the first Chapman-Enskog approximation. The collision cross sections are obtained using Gupta's method [20]. However, for e-N and e-O pairs, collision cross section models by Fertig et al. [21,22] are used. The diffusion coefficients are expressed using the formula developed by Curtiss and Hirschfelder [23]. Ambipolar diffusion is assumed for the charged species as $D_{s}^{a}=\left(1+T_{\mathrm{e}} / T_{\mathrm{tr}}\right) D_{s}$, where $D_{s}$ represents the effective diffusion coefficient of the ion species.

For chemical reactions in high-temperature air, the test gas is assumed to consist of 11 chemical species $\left(\mathrm{N}_{2}, \mathrm{O}_{2}, \mathrm{NO}, \mathrm{N}_{2}^{+}, \mathrm{O}_{2}^{+}, \mathrm{NO}^{+}, \mathrm{N}, \mathrm{O}, \mathrm{N}^{+}, \mathrm{O}^{+}\right.$, and $\left.\mathrm{e}^{-}\right)$, and 49 reactions are assumed to occur. The chemical reaction rate is determined using an Arrhenius-type form. The reaction rate coefficients are obtained from work by Park [24]. Moreover, the backward reaction rate is evaluated using the corresponding equilibrium constant. The equilibrium constants, being functions of only temperature, are calculated using the curve-fit formula in Ref. [25].

We consider the energy transfer between the following internal energy modes: translationrotation (T-R) [26], translation-vibration (T-V) [27, 28], translation-electron (T-e) [29-31], rotation-vibration $(\mathrm{R}-\mathrm{V})[26]$, rotation-electron (R-e) [32,33], and vibration-electron (Ve) [34]. The energy losses/releases for the vibrations and rotations associated with the chemical reactions reveal the dissociation energies for heavy-particle-impact reactions. These are obtained using a non-preferential dissociation model [35]. The electron energy loss/release due to electron-impact dissociation and ionization is also considered.

The electric-field equation is derived from Maxwell's equations and a generalized form of Ohm's law:

$$
\nabla \cdot(\sigma \nabla \phi)=\nabla \cdot\left(\frac{\sigma}{e n_{\mathrm{e}}} \nabla p_{\mathrm{e}}\right)
$$


where $\sigma$ represents the electric conductivity and $n_{\mathrm{e}}$ represents the electron number density. The current-density vector is calculated using the electric potential and the electron pressure:

$$
\mathbf{j}=\sigma\left(-\nabla \phi+\frac{\sigma}{e n_{\mathrm{e}}} \nabla p_{\mathrm{e}}\right) \text {. }
$$

The electric field is defined as

$$
\mathbf{E}=-\nabla \phi
$$

The Joule heating rate $S_{\text {joule }}$ is thus given by

$$
S_{\text {joule }}=\mathbf{j} \cdot \mathbf{E} .
$$

The present study uses the Abe-Kondoh-Nagano (AKN) $k-\varepsilon$ model [36], which is a lowReynolds-number two-equation turbulence model. The turbulence-transport equations include the turbulent kinetic energy $(k)$ and its dissipation rate $(\varepsilon)$.

We introduce the two following assumptions into the present radiation calculation: I) The effects of scattering are negligible, and II) the radiation field is independent of time. The radiation transport equation is calculated only in the radial direction of the cylindrical coordinate system [37]. To evaluate accurately the total radiative heat flux, it is necessary to integrate over a large number of wavelength points of $\mathrm{O}\left(10^{6}\right)$ (line-by-line calculation). However, it is too computationally expensive to integrate iteratively the radiative heat flux over all the wavelengths. In the present study, therefore, we introduce the three-band radiation model developed by Sakai and Olejniczak [6,8]. The wavelength-mean absorption coefficients and blackbody functions are evaluated a priori by classification into one of the three bands, namely, the Planck, Rosseland and Gray-gas groups. The computational cost of the three-band radiation model is significantly lower than that of the line-by-line calculation.

\section{$2.2 \quad$ Analysis Objects}

Figure 1 shows a schematic representation of the constrictor-type and segmented-type arc heaters. The $20 \mathrm{~kW} \mathrm{KUWT} \mathrm{consists} \mathrm{of} \mathrm{three} \mathrm{components,} \mathrm{namely,} \mathrm{the} \mathrm{inlet,} \mathrm{the} \mathrm{constrictor,}$ and the nozzle section. The inlet part of the $20 \mathrm{~kW} \mathrm{KUWT} \mathrm{is} \mathrm{a} \mathrm{convergence} \mathrm{nozzle} \mathrm{with}$ a half angle of $45^{\circ}$. The length and diameter of the constrictor are $15.6 \mathrm{~mm}$ and $5.0 \mathrm{~mm}$, respectively. The nozzle geometry is conical with a half angle of $15^{\circ}$ and the nozzle-exit diameter is $100 \mathrm{~mm}$. The cathode tip is located at the constrictor entrance. In the $750 \mathrm{~kW}$ JXWT, the constrictor section is approximately $390 \mathrm{~mm}$ in length and $25 \mathrm{~mm}$ in diameter, and the throat diameter is $5 \mathrm{~mm}$. The nozzle of the $750 \mathrm{~kW}$ JXWT is conical with a half angle of $15^{\circ}$ and the nozzle-exit diameter is $115 \mathrm{~mm}$. The constrictor section of the $20 \mathrm{MW}$ AHF is approximately $2300 \mathrm{~mm}$ in length and $60 \mathrm{~mm}$ in diameter. Its throat is $39 \mathrm{~mm}$ in diameter and $50 \mathrm{~mm}$ in length. The nozzle of the $20 \mathrm{MW}$ AHF is also conical, with a half angle of $8^{\circ}$, and the nozzle-exit diameter is $304 \mathrm{~mm}$. An arc discharge is generated on the cathode and attaches to the anode surface. The test gas injected subsonically into the inlet part is strongly heated by the arc discharge in the constrictor and expands through the nozzle. The high-enthalpy flow is then exhausted to the vacuum chamber in the tunnel system.

\subsection{Boundary Conditions}

The flow is subsonically injected at the inlets of the arc heaters. The static pressure at the inlet is extrapolated from the interior point, whereas the other flow properties are calculated 

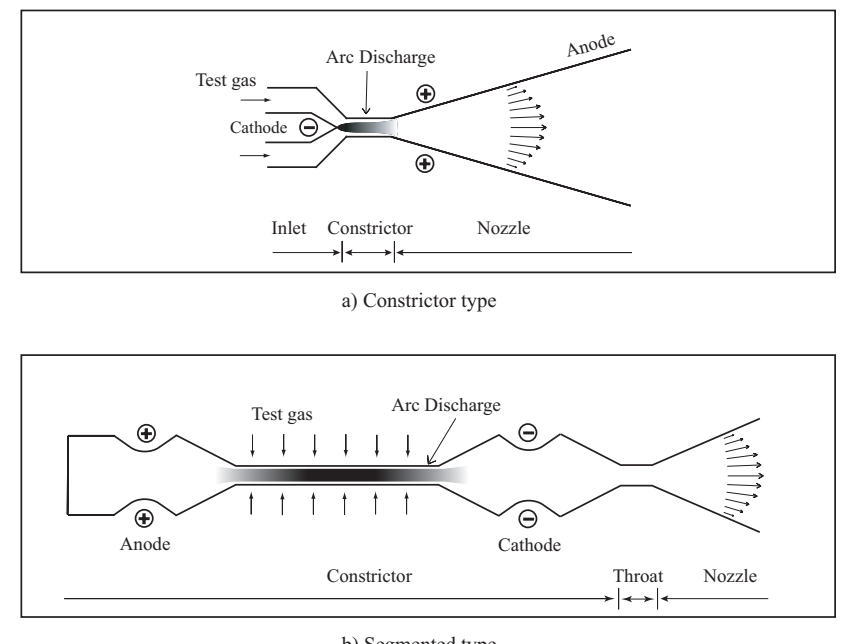

Segmented type

Figure 1: Schematic representation of arc heaters

from the specified total temperature and the mass-flow rate. At the nozzle exit, all the flow properties are determined by the zeroth extrapolation, because the flow is supersonic in most regions except for the boundary layer close to the nozzle wall. The non-slip condition for the velocity and the non-catalytic condition for the mass concentration are imposed at the electrode walls. There is assumed to be no pressure gradient normal to the wall. The wall temperature is determined by the radiative equilibrium equation. Note that, in this study, the wall temperature is controlled so that it remains below $1000 \mathrm{~K}$. An axisymmetric condition is imposed along the symmetry axis.

No current is allowed to pass through the inlet or the nozzle exit. An equipotential condition is set at the electrode wall: $V=0$ at the cathode surface, and $V=V_{0}$ at the anode surface. In the computational procedure, the arc voltage is determined so that the total current in the flow field is equal to the input current. That is, the arc voltage $V^{n+1}$ at the $(n+1)$ th time step can be calculated from the input current $I$, the total current $I^{n}$ in the flow field, and the arc voltage $V^{n}$ at the $n$th step, which is expressed as $V^{n+1}=$ $V^{n}+\Delta V^{n}, \quad \Delta V^{n}=\omega\left(\frac{I}{I^{n}}-1\right) V^{n}$, where $\omega$ is the relaxation parameter.

\subsection{Calculation Conditions}

In the present calculation, the input parameters for the $750 \mathrm{~kW}$ JXWT, $20 \mathrm{MW}$ AHF, and $20 \mathrm{~kW}$ KUWT are the mass-flow rate $(\dot{m})$, the arc current $(I)$, and the test gas. The test gas is air for the $750 \mathrm{~kW}$ JXWT and $20 \mathrm{MW}$ AHF, whereas nitrogen is considered for the 20 kW KUWT.

Figures 2(a) and 2(b) respectively show the computational domains and grids system for the $750 \mathrm{~kW}$ JXWT and $20 \mathrm{~kW}$ KUWT, although the computational grid system for the 20 MW AHF is not shown here. Note that the grids of the $750 \mathrm{~kW}$ JXWT shown in Fig. 2(a) are partially omitted for clarity. The computational grid nodes are set to 265 (axial) $\times 45$ (radial) for the $750 \mathrm{~kW} \mathrm{JXWT} \mathrm{and} 250$ (axial) $\times 60$ (radial) for the $20 \mathrm{MW} \mathrm{AHF}$. The coordinate origin is set at the throat inlet on the center axis for both the $750 \mathrm{~kW}$ JXWT and the $20 \mathrm{MW}$ AHF. In the $750 \mathrm{~kW}$ JXWT, the constrictor section is variable from $130 \mathrm{~mm}$ to $390 \mathrm{~mm}$, because it is composed of assemblies of the segmented disks called "packs." One pack consists of 13 disks and is $130 \mathrm{~mm}$ in length. In the present study, 2-pack and 3-pack 
cases are numerically simulated. On the other hand, the computational grid nodes for the $20 \mathrm{~kW} \mathrm{KUWT}$ are set to 155 (axial) $\times 55$ (radial). The origin is located at the cathode tip $(x=0 \mathrm{~mm})$.

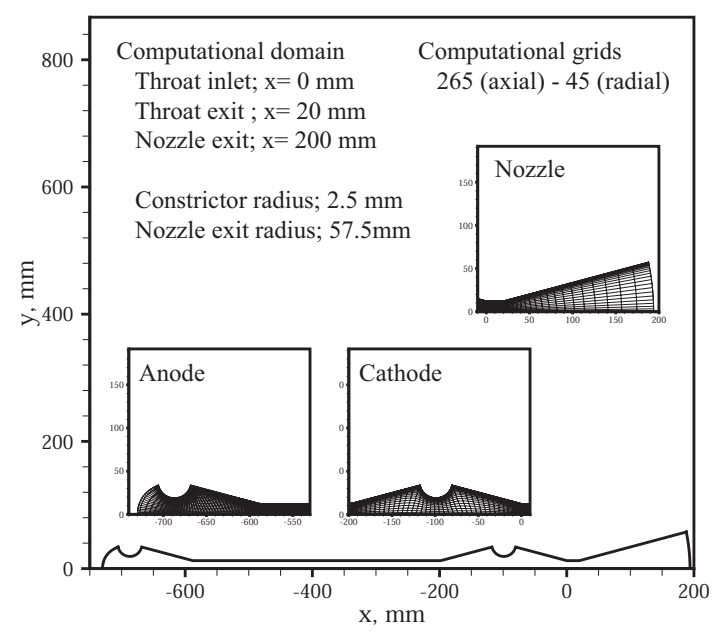

(a) $750 \mathrm{~kW}$ JXWT (3-pack)

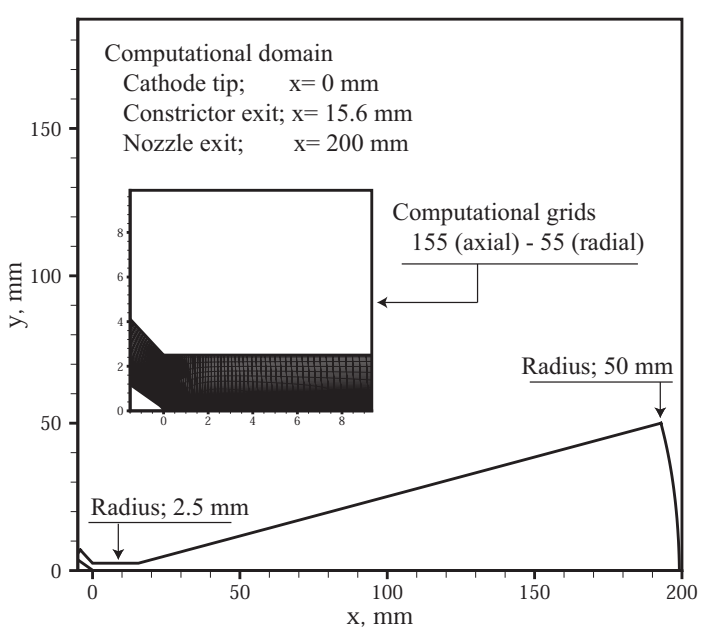

(b) $20 \mathrm{~kW}$ KUWT

Figure 2: Computational domains and grids for $750 \mathrm{~kW}$ JXWT and $20 \mathrm{~kW}$ KUWT

\section{Results and Discussion}

\subsection{Arc-Heated Flow in Constrictor Section}

\subsubsection{Heat flux on constrictor wall}

In the constrictor section, energy is input into the flow by the arc discharge. To predict an arc-jet in the vacuum chamber accurately, it is important to use a sufficiently detailed model and validate the plasma behavior, the energy cascade from the arc discharge to the flow field, and the energy loss by radiation and turbulence in the constrictor of the arc-heating facility. Hence, the predicted flow properties in the constrictor need to be compared with the measured properties. However, few experimental data have been collected to date because of the difficulties of measurement. The distribution of the electronic excitation temperature and the heat flux in the constrictor section were obtained recently by Mizuno et al. [18] using a custom segmented disk technique for the $750 \mathrm{~kW}$ JXWT. An observation window and a heat flux sensor were fixed on the custom segment. An optical fiber through the window was used to observe the emission spectra of the arc column. Radiation intensity of the atomic emission lines such as $\mathrm{N}$ and $\mathrm{O}$ was measured for determining the electronic excitation temperature by using the Boltzmann plot method. The heat flux sensor was used to measure the total heat flux that corresponded to the sum of the radiative and convective heat fluxes on the segmented wall. These measurements were performed for the 2-pack and 3-pack conditions of the $750 \mathrm{~kW}$ JXWT.

Figure 3 shows a comparison of the electronic excitation temperature profiles on the segmented wall in the case of the 3-pack of the $750 \mathrm{~kW}$ JXWT. The dashed lines show the 
experimental results, and the solid lines show the computational results. In the present calculation, the electronic excitation temperature is assumed to be equal to the electron temperature $\left(T_{\mathrm{ex}}=T_{\mathrm{e}}\right)$. The electronic excitation temperature is slightly underestimated compared with the measured temperature. Although a qualitatively good agreement is shown, the computational results cannot reproduce the temperature increase found in the middle region $(x=-400$ to $-300 \mathrm{~mm})$ of the constrictor. As shown in the temperature profiles for each current condition (Fig. 3), the temperature in the arc column is insensitive to the Joule heating rate because most of the energy input by the discharge is spent on the chemical reactions. Hence, it is difficult to explain the large increase in temperature based on only the increase in the Joule heating rate. Because the flow velocity in the discharge section is low and the flow density is relatively high, the collision frequency between particles is high. As confirmed by the numerical predictions given in Ref. [13], the flow is close to the thermal equilibrium state in this JXWT case. However, it is possible that some peculiar nonequilibrium mechanism is present in the energy cascade process of the arc column brought about by the discharge. The energy generated by the discharge is first input into the electron excitation energy or the electron energy mode and then transferred to the rotational and vibrational energy modes. The translational energy mode is finally excited in the energy cascade process. Thus far, a multitemperature model has been developed using a considerable amount of experimental data from shock tube facilities. However, the energy-transfer process of this model is different from that in an arc-heating facility. Although, in the present chemical system, we take into account 11 species and 49 reactions for air, a relatively detailed discharge model, e.g., a state-to-state model [3], may need to be introduced.

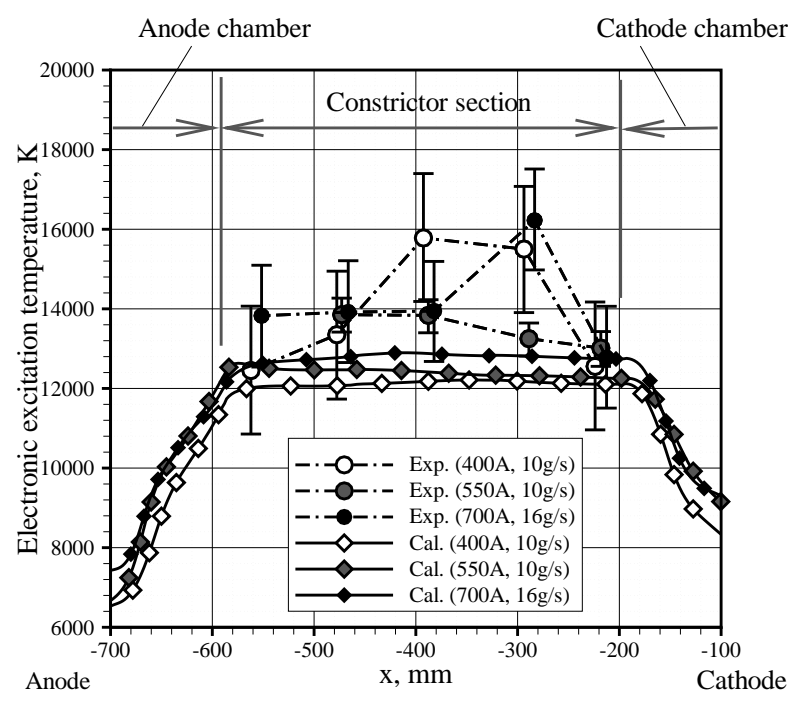

Figure 3: Comparison of electronic excitation temperature in the constrictor of the $750 \mathrm{~kW}$ JXWT (3-pack)

The profiles of the total heat flux on the wall of the $750 \mathrm{~kW}$ JXWT in the case of $I=500$ A and $\dot{m}=10.0 \mathrm{~g} / \mathrm{s}$ are shown in Fig. 4. On the other hand, Fig. 5 shows a comparison of the experimental and computational results of the heat fluxes on the segmented wall in the case of the 2-pack condition of the $750 \mathrm{~kW}$ JXWT. It can be seen from Fig. 5 that the computational results are considerably underestimated. This is the natural consequence of the results for the electronic excitation temperature. As mentioned earlier in the present section, 
the electron temperature is high in the constrictor section. In general, the radiative heat flux $\left(q_{\mathrm{rad}}\right)$ is strongly affected by the temperature, and the radiation rapidly increases when the temperature exceeds $10000 \mathrm{~K}$. As seen in Fig. 4, in the constrictor section, the radiative heat flux is dominant, whereas the convective heat flux $\left(q_{\text {con }}\right)$ becomes high in both the anode and the cathode chambers. As shown in Fig. 3, the electronic excitation temperature is underestimated in the constrictor section. This directly leads to the underestimation of the radiative heat flux. Of course, it is important to calculate accurately the heat flux on the wall for reproducing the energy loss in the arc-heated flow. If prediction of the electronic excitation temperature is improved in the future, the heat flux prediction will show considerably better agreement with the experimental results.

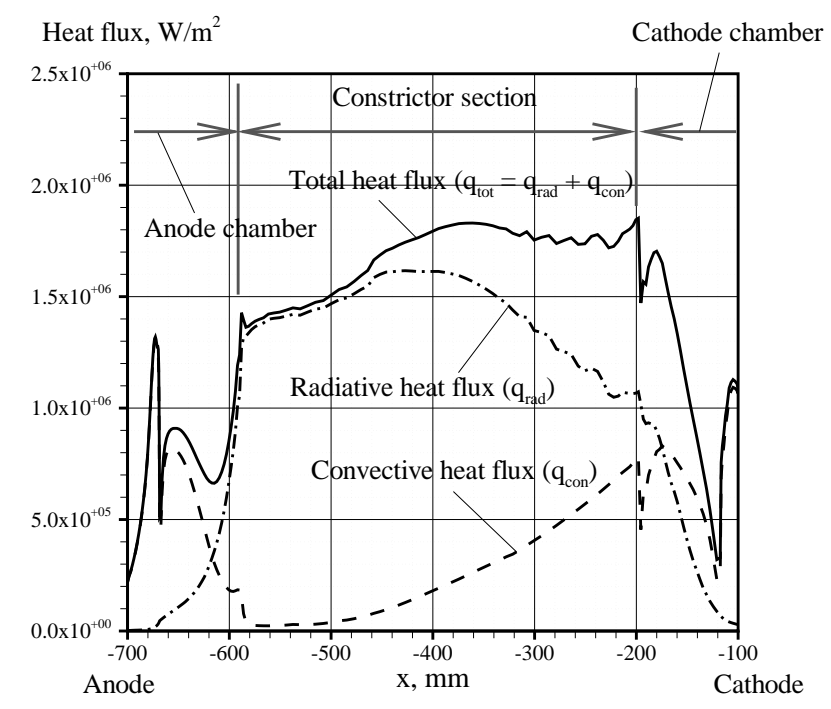

Figure 4: Axial profiles of total heat fluxes on the wall in the constrictor of the $750 \mathrm{~kW}$ JXWT (3-pack) in the case of $I=500 \mathrm{~A}$ and $\dot{m}=10.0 \mathrm{~g} / \mathrm{s}$

In the case of the present computational grids in the constrictor and nozzle for the 750 kW JXWT, the resolution is sufficient. On the other hand, the grids system in the cathode chamber appears to be relatively coarse for the RANS technique. Although discussion about the discharge behavior is mainly focused on electronic excitation temperature and radiative heat flux in the constrictor section, turbulence is an important mechanism in the transfer of heat and momentum in the cathode chamber and throat section. Thus, if more detailed investigation for turbulence is needed, calculations with a finer grid system are required.

\subsubsection{Arc voltage}

As shown in our previous research [13], the arc voltage is considerably underestimated in all the cases, and discrepancies of 300-400 V are observed in the case of the $750 \mathrm{~kW}$ JXWT, although the general trend of the predicted arc voltage for the total current or the mass-flow rate shows good agreement with the experimentally obtained relation. As shown in Fig. 4, the heat loss of the facility is mainly attributed to the radiative heat flux on the constrictor wall. In the present computation, the radiative heat flux appears to be underestimated, and hence, it is reasonable to assume that the heat loss is also underestimated. If the heat loss increases, the arc voltage consequently increases, and the computed current becomes equal 


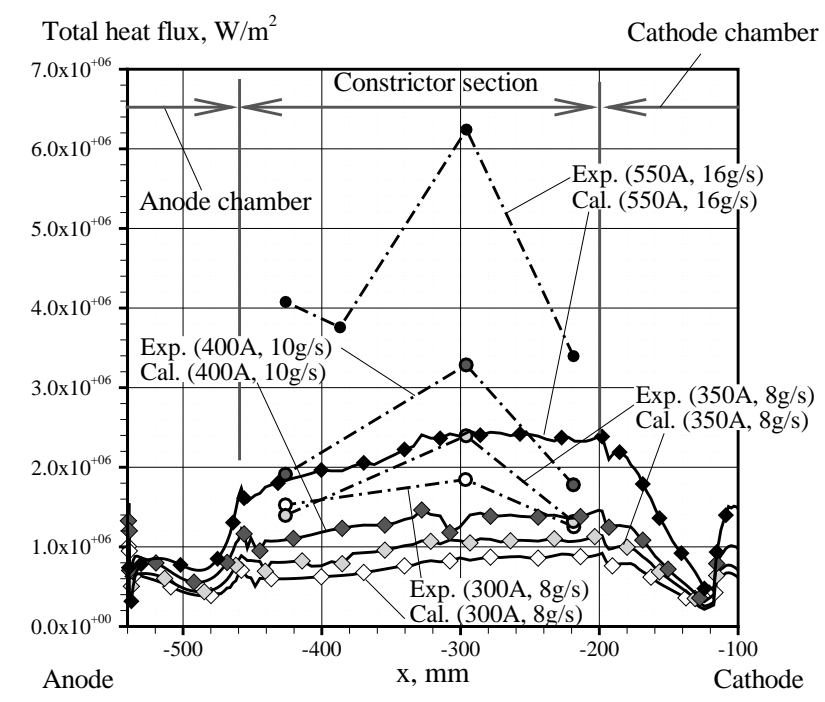

Figure 5: Comparison of total heat flux on the wall in the constrictor of the $750 \mathrm{~kW}$ JXWT (2-pack)

to the input current. Another major reason for the underestimation of the arc voltage is the electrical sheath on the electrode surfaces. This sheath causes a large potential drop [38]. Although the arc voltage correlates closely with the input energy, it is possible that the net energy in the flow field is insensitive to the discharge voltage. In fact, in the case of the 20 kW KUWT simulation [12], the flow enthalpy distribution at the nozzle exit showed a smaller difference between the computational results with/without the radiation model, although the predicted arc voltage and the impact pressure were improved.

In the simulations of the $20 \mathrm{MW}$ AHF case [13], it was reported that the calculated voltage tends to increase with an increase in the mass-flow rate, whereas it is insensitive to a change in the electric current. As in the $750 \mathrm{~kW}$ JXWT case, in the $20 \mathrm{MW}$ AHF case, the calculated arc voltage is underestimated compared with the measured voltage by approximately 500-1000 V.

In the $20 \mathrm{~kW}$ KUWT case, because the arc column is highly confined by the cold gas for a relatively high mass-flow rate, the radius of the arc column decreases. A small hightemperature region causes a decrease in the electric conductivity. Thus, as the mass-flow rate increases, the arc voltage also increases. In Ref. [12], it was reported that the predicted arc voltage is considerably lower than the measured arc voltage in all the cases. This is expected to be attributed to the electrical sheath on the electrode surface. In fact, a potential drop of approximately $10 \mathrm{~V}$ has been reported in the numerical simulations of a hydrogen arcjet $[39,40]$.

\subsection{Free-Jet Properties in Vacuum Chamber}

\subsubsection{Enthalpy}

In the case of the $750 \mathrm{~kW}$ JXWT, as shown in the previous study [13], the computational results of the flow enthalpy at the nozzle exit generally reproduced the trend shown in the experimental data, although the numerical predictions contained some underestimations. The flow enthalpy and the arc voltage appear to correlate strongly with each other at a 
constant current. However, the results show that the discrepancies between the measured and computed flow enthalpies were relatively small, whereas those for the arc voltage were large. Based on the results of the flow enthalpy, the net energy input into the flow field in the facility was expected to be predicted accurately by the present analysis model. Hence, in the present model, it is possible that the remaining energy that was never input into the gas (e.g., potential drop near the electrodes and the heat loss on the wall) was inaccurately predicted. From this point of view, it is reasonable to conclude that the computed radiative heat flux on the wall strongly underestimates the measured radiative heat flux, as shown in Fig. 5. On the other hand, the computational results for the mass-averaged enthalpy show good agreement with the experimental data under various operating conditions in the case of the $20 \mathrm{MW}$ AHF [13].

Comparisons of the impact pressure and heat flux at the stagnation point of a probe are shown in Ref. [13]. It was confirmed in that study that the computational results quantitatively agreed with the experimental data, although they are slightly overestimated compared with the measured results under the condition of a low mass-flow rate. Both the pressure and the heat flux correlates with the local flow enthalpy, according to work by Pope [41]. Our results indicate that the present analytical model is able to reproduce the enthalpy of a free jet for various types of arc heaters.

\subsubsection{Temperatures}

Figure 6 shows a comparison of the rotational temperature $60 \mathrm{~mm}$ downstream from the nozzle exit in the $750 \mathrm{~kW}$ JXWT under several current conditions. The experimental data [14] were obtained by spectroscopic analysis of molecular band emission. The rotational temperature was determined by obtaining nitric oxide (NO)-band spectra (i.e., $\delta(0,1), \delta(0,2)$, $\delta(0,3), \gamma(0,0)$, and $\gamma(0,1))$. Comparison of the rotational temperature shows quantitatively good agreement. It is confirmed that the temperatures are completely separated and that the plasma flow is strong at thermal nonequilibrium. The electron temperature decreases with an increase in the input current. Under the high-current condition, the arc-heated flow tends to contain many ion species and electrons because of severe dissociation and ionization in the constrictor section. As the number of electrons increases, the internal energy transfer between the electron energy and the other energy modes proceeds rapidly. Consequently, the electron temperature tends to be equilibrated with the temperatures of the other internal energy modes.

Figure 7 shows a comparison of the radial distribution of the translational temperature $357 \mathrm{~mm}$ downstream from the nozzle exit in the $20 \mathrm{MW}$ AHF when $I=1160 \mathrm{~A}$ and $\dot{m}=100$ $\mathrm{g} / \mathrm{s}$. The experimental data were obtained using the LIF technique [17]. The computational results for the translational temperature show good agreement with the experimental data in the $20 \mathrm{MW}$ AHF case, although slight underestimations were observed in the calculated temperature.

In the case of the $20 \mathrm{~kW} \mathrm{KUWT}$, a spectroscopic measurement was conducted, and NOband spectra in the wavelength range of 230-260 $\mathrm{nm}$ were obtained from Ref. [2]. Moreover, the NO rotational temperatures were identified using a curve-fitting method for the NO $\gamma(0,1)$ spectra with SPRADIAN [42] to calculate the spectral lines. Figure 8 shows a comparison of the rotational temperature at the nozzle exit. The predicted translational, vibrational, and electron temperatures are also included in this figure. The predicted rotational temperature shows qualitative agreement, although a $30 \%$ overestimation was observed at a high current. Comparing the cases of the $750 \mathrm{~kW}$ JXWT and the $20 \mathrm{MW}$ AHF, the relaxation time between 
the translational and rotational energy modes slows because of low density and high velocity in the nozzle section of the $20 \mathrm{~kW}$ KUWT. The rotational energy is supplied with chemical energy by the recombination reaction of atomic molecules (reverse reaction of heavy-particle impact dissociation) in the expansion process, and the rotational temperature increases. In the present calculations, this mechanism is expressed with the non-preferential model, which is relatively simple and has low-computational cost. To reduce the error in the case of the $20 \mathrm{~kW}$ KUWT, a more detailed model may need to be introduced.

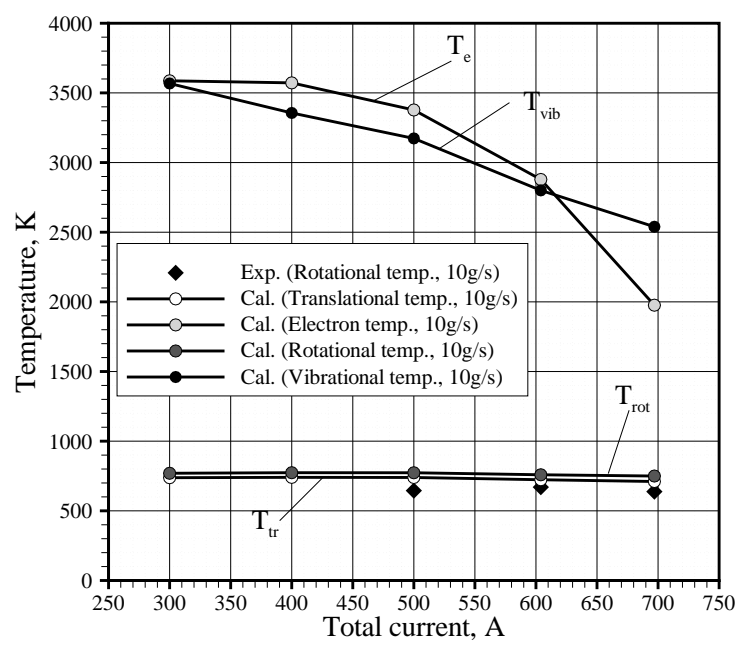

Figure 6: Comparison of rotational temperature $60 \mathrm{~mm}$ downstream from the nozzle exit of the $750 \mathrm{~kW}$ JXWT in the case of $\dot{m}=10.0 \mathrm{~g} / \mathrm{s}$

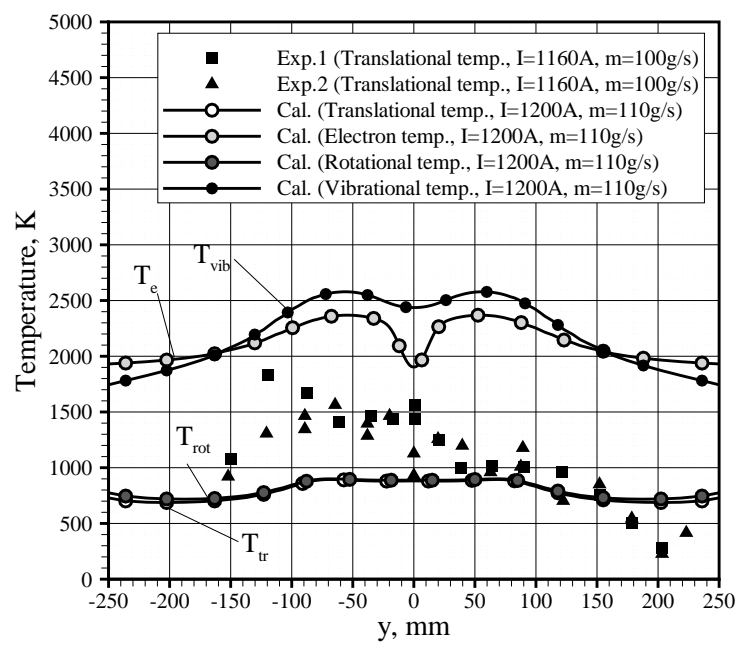

Figure 7: Comparison of translational temperature $357 \mathrm{~mm}$ downstream from the nozzle exit of the $20 \mathrm{MW}$ AHF in the case of $I=1160 \mathrm{~A}$ and $\dot{m}=100 \mathrm{~g} / \mathrm{s}$ 


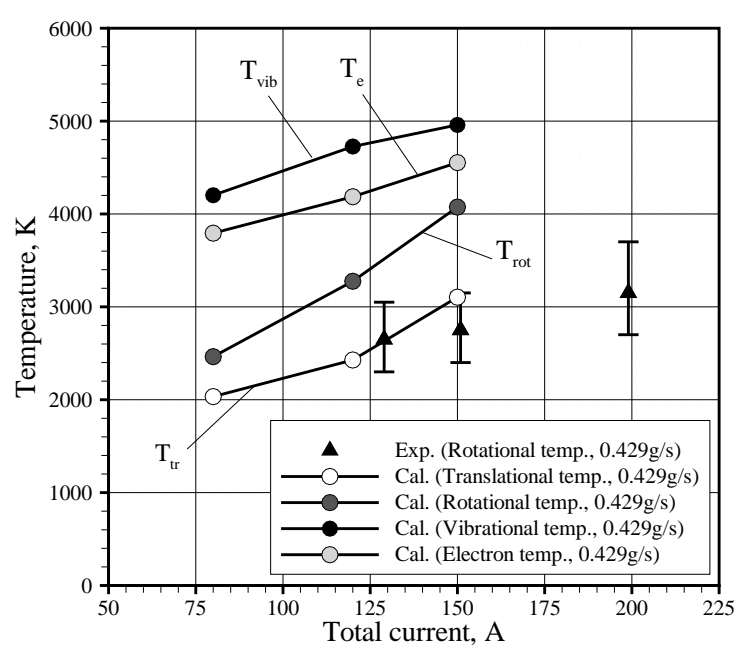

Figure 8: Comparison of rotational temperature at the nozzle exit of the 20 KUWT

\subsubsection{Number density}

A radial profile comparison of the density of atomic nitrogen $100 \mathrm{~mm}$ downstream from the nozzle exit in the $750 \mathrm{~kW}$ JXWT is shown in Fig. 9. The mass-flow rate was set to 10.0 $\mathrm{g} / \mathrm{s}$ for both of the current conditions of $I=300 \mathrm{~A}$ and $I=700 \mathrm{~A}$. The experimental data were obtained by the LIF technique $[16,43]$. The computational results of the low-current condition show good agreement with the experimental data.

Figure 10 shows a comparison of the radial distribution of the number density of atomic nitrogen $357 \mathrm{~mm}$ downstream from the nozzle exit in the $20 \mathrm{MW}$ AHF when $I=1160 \mathrm{~A}$ and $\dot{m}=100 \mathrm{~g} / \mathrm{s}$ [17]. In the core-flow region near the centerline $(y=0 \mathrm{~mm})$, the predicted number density shows good agreement with the experimental data, whereas the predicted number density outside the core decreases to less than the measured number density. In the present calculation, it is possible that the mass diffusion in the radial direction is not as active as that in the real world.

In the constrictor section of large-scale arc heaters such as the $750 \mathrm{~kW}$ JXWT and the $20 \mathrm{MW}$ AHF, turbulence plays a significant role. The momentum and heat transfer mechanisms caused by turbulence are considered here, whereas the turbulent mass transfer is not introduced in this model. Moreover, it is possible that the contributions of the rarefied gas effects in the vacuum chamber have a significant influence on the formation of the flow property distributions; here, for the sake of simplicity, the flow is assumed to be continuous. In the present study, a relatively simple model of a chemical reaction system is used, and the effect of the electronically metastable states of atoms and molecules is neglected. As discussed in Refs. [44,45], the behavior of the chemical reaction could change if the electronically metastable states of molecules and atoms are considered. In the present calculations, thermal equilibrium between the electron energy and electronic excitation energy modes is assumed, whereas this approximation may be insufficient for the freejet in the vacuum chamber. For simplicity and to reduce computational cost, we neglected the contribution of electronically metastable states and then considered 11 species and 49 reactions in the present chemical reaction system [25]. A more detailed discussion of this issue is left for future studies. 


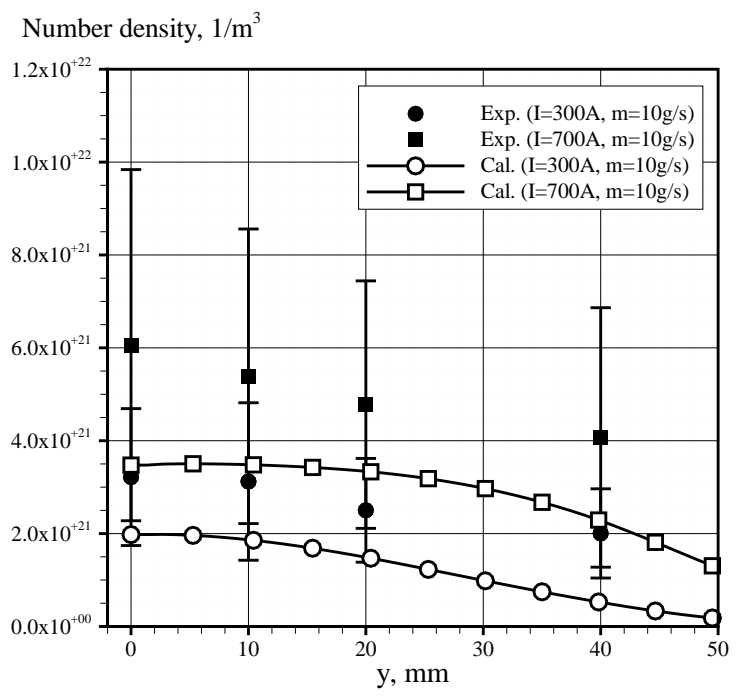

Figure 9: Comparison of number density of atomic nitrogen $100 \mathrm{~mm}$ downstream from the nozzle exit in the $750 \mathrm{~kW}$ JXWT in the cases of $I=300 \mathrm{~A}$ and $\dot{m}=10.0 \mathrm{~g} / \mathrm{s}$ and $I=700$ A and $\dot{m}=10.0 \mathrm{~g} / \mathrm{s}$

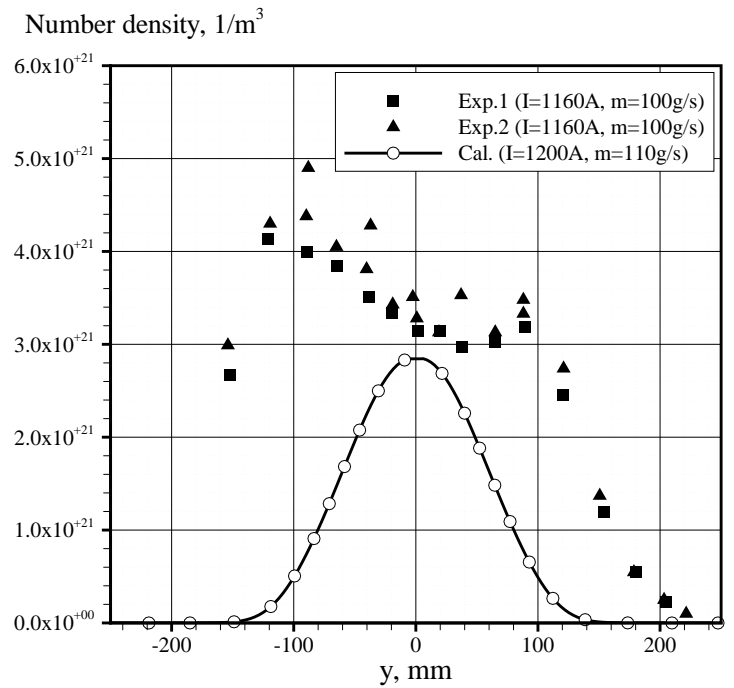

Figure 10: Comparison of number density of atomic nitrogen $357 \mathrm{~mm}$ downstream from the nozzle exit in the $20 \mathrm{MW}$ AHF for the case of $I=1160 \mathrm{~A}$ and $\dot{m}=100 \mathrm{~g} / \mathrm{s}$

\section{Conclusions}

Numerical simulations of plasma flows were performed for three types of arc-heating facilities, namely, the JAXA $750 \mathrm{~kW}$ arc-heated wind tunnel (JXWT), the NASA $20 \mathrm{MW}$ aerodynamic heating facility (AHF) and the Kyushu University $20 \mathrm{~kW}$ arc-heated wind tunnel (KUWT). To validate the present numerical model, the calculated data were compared with the experimental data, which included the mass-averaged enthalpy, the translational and rotational temperatures, and the number density of atomic nitrogen. Moreover, the present discharge 
model was validated using the measured profiles of the electronic excitation temperature and the heat flux on the segmented wall in the constrictor of the $750 \mathrm{~kW}$ JXWT. Through these comparisons, the arc-heated flow behaviors of the abovementioned arc-heating facilities were discussed in detail. Good agreement with the experimental data was generally obtained, and the present model may appropriately predict the free-jet properties. Thus, an integration analysis model was developed to simulate the arc-heated flow fields for various-scale facilities.

On the other hand, the calculated number density of atomic nitrogen was underestimated in the outer region of the core flow. A mass diffusion mechanism may be present in the radial direction that is not considered here, such as a strong turbulent mass transfer in the constrictor section and/or rarefied gas effects in the vacuum chamber. Moreover, in the case of the $20 \mathrm{~kW}$ KUWT, the predicted rotational temperature in the vacuum chamber overestimated the measured temperature at high current. The predicted electronic excitation temperature and the heat flux in the constrictor section of the $750 \mathrm{~kW}$ JXWT were underestimated compared with the measured electronic excitation temperature and heat flux, although qualitatively good agreements were found. It was clarified that, in order to describe the energy-transfer mechanism by discharge and expansion, more detailed discharge models may be required. The possible contributions of such a model may need to be examined to develop the calculation further in future research.

\section{Acknowledgments}

The computation was mainly carried out using the computer facilities at Research Institute for Information Technology, Kyushu University.

\section{References}

[1] C. Park and S.-H. Lee. "Validation of Multitemperature Nozzle Flow Code". Journal of Thermophysics and Heat Transfer, Vol. 9, No. 1, pp. 9-16, Jan.-Mar. 1995.

[2] K. Abe, T. Kameyama, H. Kihara, M. Nishida, K. Ito, and H. Tanno. "Computation and Experiment of Nonequilibrium Nozzle Flow of Arc-heated Air". Journal of Thermophysics and Heat Transfer, Vol. 19, No. 4, pp. 428-434, 2005.

[3] G. Colonna and M. Capitelli. "Boltzmann and Master Equations for Magnetohydrodynamics in Weakly Ionized Gases". Journal of Thermophysics and Heat Transfer, Vol. 22, No. 3, pp. 414-423, July-Sept. 2008.

[4] K. H. Kim, O. H. Rho, and C. Park. "Navier-Stokes Computation of Flows in Arc Heaters". Journal of Thermophysics and Heat Transfer, Vol. 14, No. 2, pp. 250-258, April-June 2000.

[5] T. Sakai, K. Sawada, and M. Mitsuda. "Application of Planck-Rosseland-Gray Model for High-Enthalpy Arc Heaters". Journal of Thermophysics and Heat Transfer, Vol. 15, No. 2, pp. 176-183, April-June 2001.

[6] T. Sakai and J. Olejniczak. "Improvements in a Navier-Stokes Code for Arc Heater Flows". AIAA Paper 2003-3782, 2003. 
[7] J.-P. Lee, C. Kim, and K.-H. Kim. "Accurate Computations of Arc-Heater Flows Using Two-Equation Turbulence Models". Journal of Thermophysics and Heat Transfer, Vol. 21, No. 1, pp. 67-76, Jan.-Mar. 2007.

[8] T. Sakai. "Computational Simulation of High-Enthalpy Arc Heater Flows". Journal of Thermophysics and Heat Transfer, Vol. 21, No. 1, pp. 77-85, Jan.-Mar. 2007.

[9] Y. Takahashi, H. Kihara, and K. Abe. "Numerical Investigation of Nonequilibrium Plasma Flows in Constrictor- and Segmented-Type Arc Heaters". Journal of Thermophysics and Heat Transfer, Vol. 24, No. 1, pp. 31-39, 2010.

[10] T. Matsuzaki, K. Ishida, Y. Watanabe, K. Miho, H. Itagaki, and T. Yoshinaka. "Constructions and Characteristics of the $750 \mathrm{~kW}$ Arc Heated Wind Tunnel". NAL TM-760, National Aerospace Laboratory, Chofu, Japan, 2001.

[11] T. M. Hightower, J. A. Balboni, C. L. M. Donald, K. F. Anderson, and E. R. Martinez. "Enthalpy by Energy Balance for Aerodynamic Heating Facility at NASA Ames Research Center Arc Jet Complex". In Proceedings of the 48th International Instrumentation Symposium, Vol. 420, Instrumentation, Systems, and Automation Society, San Diego, CA, USA, 2002.

[12] Y. Takahashi, H. Kihara, and K. Abe. "The Effects of Radiative Heat Transfer in Arc-Heated Nonequilibrium Flow Simulation". Journal of Physics D: Applied Physics, Vol. 43, No. 18, p. 185201, 2010.

[13] Y. Takahashi, H. Kihara, and K. Abe. "Turbulence and Radiation Behaviours in LargeScale Arc Heaters". Journal of Physics D: Applied Physics, Vol. 44, No. 8, p. 085203, 2011.

[14] Y. Watanabe, K. Ishida, and H. Shirai. "Spectroscopic Analysis of NO Band Emissions from Arc-Heated Air Flows in a $750 \mathrm{~kW}$ Arc-Heated Wind Tunnel". NAL TR-1417, National Aerospace Laboratory, Chofu, Japan, 2000.

[15] H. Takayanagi, M. Mizuno, K. Fujii, T. Suzuki, and K. Fujita. "Arc Heated Wind Tunnel Flow Diagnostics using Laser-Induced Fluorescence of Atomic Species". AIAA Paper 2009-1449, 2009.

[16] H. Takayanagi, M. Mizuno, K. Fujii, T. Suzuki, and K. Fujita. "Arc Wind Tunnel Flow Characterization Measured by Laser-Induced Fluorescence of Atomic Species". AIAA Paper 2009-4241, 2009.

[17] J. H. Grinstead, D. M. Diver, and G. A. Raiche. "Radial Profiles of Arcjet Flow Properties Measured with Laser-Induced Fluorescence of Atomic Nitrogen". AIAA Paper 2003-400, 2003.

[18] M. Mizuno, K. Ishida, T. Matsuzaki, T. Ito, and Y. Watanabe. "Spectral and Heating Characteristics in the Arc Column of the 0.75 MW Arc-Heated Wind Tunnel". In Proceedings of 23th International Symposium on Space Technology and Science, ISTS 2002-e-4, Matsue, Shimane, Japan, May 26 - June 22002.

[19] M. W. Winter, D. K. Prabhu, G. A. Raiche, I. Terrazas-Salinas, and F. C. L. Hui. "Emission Spectroscopic Measurement with an Optical Probe in the NASA Ames IHF Arc Jet Facility". AIAA Paper 2012-1016, 2012. 
[20] R. N. Gupta, J. M. Yos, R. A. Thompson, and K.-P. Lee. "A Review of Reaction Rates and Thermodynamic and Transport Properties for an 11-Species Air Model for Chemical and Thermal Nonequilibrium Calculations to 30000 K". NASA RP-1232, Aug. 1990.

[21] M. Fertig, A. Dohr, and H.-H. Frühaufu. "Transport Coefficients for High-Temperature Nonequilibrium Air Flows". AIAA Paper 98-2937, 1998.

[22] M. Fertig, A. Dohr, and H.-H. Frühauf. "Transport Coefficients for High-Temperature Nonequilibrium Air Flows". Journal of Thermophysics and Heat Transfer, Vol. 15, No. 2, pp. 148-156, April-June 2001.

[23] C. F. Curtiss and J. O. Hirschfelder. "Transport Properties of Multicomponent Gas Mixture". Journal of Chemical Physics, Vol. 17, No. 6, pp. 550-555, June 1949.

[24] C. Park. "Assessment of a Two-Temperature Kinetic Model for Dissociating and Weakly Ionizing Nitrogen". Journal of Thermophysics and Heat Transfer, Vol. 2, No. 1, pp. 8-16, Jan.-March 1988.

[25] C. Park. Nonequilibrium Hypersonic Aerothermodynamics. Wiley, New York, 1990.

[26] C. Park. "Rotational Relaxation of $\mathrm{N}_{2}$ Behind a Strong Shock Wave". Journal of Thermophysics and Heat Transfer, Vol. 18, No. 4, pp. 527-533, Oct.-Dec. 2004.

[27] R. C. Millikan and D. R. White. "Systematics of Vibrational Relaxation". The Journal of Chemical Physics, Vol. 39, No. 12, pp. 3209-3213, Dec. 1963.

[28] C. Park. "Problems of Rate Chemistry in the Flight Regimes of Aeroassisted Orbital Transfer Vehicles". AIAA Paper 84-1730, 1984.

[29] J. P. Appleton and K. N. C. Bray. "The Conservation Equations for a Nonequilibrium Plasma". Journal of Fluid Mechanics, Vol. 20, No. 4, pp. 659-672, June 1964.

[30] M. Mitchner and C. H. K. Jr. Partially Ionized Gases. Wiley, New York, 1973.

[31] J.-H. Lee and D. R. White. "Thermal Design of Aeroassisted Orbital Transfer Vehicles". In H. F. Nelson, editor, Basic Governing Equations for the Flight Regimes of Aeroassisted Orbital Transfer Vehicles (Progress in Astronautics and Aeronautics), Vol. 96, pp. 3-53. AIAA, New York, 1985.

[32] M. Nishida and M. Matsumoto. "Thermochemical Nonequilibrium in Rapidly Expanding Flows of High-Temperature Air". Zeitschrift für Naturforcuhung, Teil A: Physik, Physikalische Chemie, Kosmophysik, Vol. 52, No. 4, pp. 358-368, 1997.

[33] S. S. Lazdinis and S. L. Petrie. "Free Electron and Vibrational Temperature Nonequilibrium in High Temperature Nitrogen". Physics of Fluids, Vol. 17, No. 8, pp. 1539-1546, Aug. 1974.

[34] J.-H. Lee. "Electron-Impact Vibrational Relaxation in High-Temperature Nitrogen". Journal of Thermophysics and Heat Transfer, Vol. 7, No. 3, pp. 399-405, July-Sept. 1993.

[35] P. A. Gnoffo, R. N. Gupta, and J. L. Shinn. "Conservation Equations and Physical Models for Hypersonic Air Flows in Thermal and Chemical Nonequilibrium". NASA TP-2867, Feb. 1989. 
[36] K. Abe, T. Kondoh, and Y. Nagano. "A New Turbulence Model for Predicting Fluid Flow and Heat Transfer in Separating and Reattaching Flows - I. Flow Field Calculations". International Journal of Heat and Mass Transfer, Vol. 37, No. 1, pp. 139-151, 1994.

[37] A. S. Kesten. "Radiant Heat Flux Distribution in a Cylindrically-Symmetric Nonisothermal Gas with Temperature-Dependent Absorption Coefficient". J. Quant. Spectrosc. Radiat. Transfer, Vol. 8, pp. 419-434, 1968.

[38] Y. Takahashi, H. Kihara, and K. Abe. "Improvement of Potential Drop Predictions for Plasma Wind Tunnels by Cathode Sheath". Journal of Thermophysics and Heat Transfer, Vol. 26, No. 83, pp. 540-544, July-September 2012.

[39] K. Fujita and Y. Arakawa. "Performance Computation of a Low-Power Hydrogen Arcjet". Journal of Propulsion and Power, Vol. 15, No. 1, pp. 144-150, Jan.-Feb. 1999.

[40] J. Lu, H. Krier, R. L. Burton, and K. D. Goodfellow. "Cathode Sheath Voltage Models for Hydrazine Arcjets". Journal of Thermophysics and Heat Transfers, Vol. 12, No. 2, pp. 230-238, April-June 1998.

[41] R. B. Pope. "Measurements of Enthalpy in Low-Density Arc-Heated Flows". AIAA Journal, Vol. 6, No. 1, pp. 103-110, January 1968.

[42] K. Fujita and T. Abe. "SPRADIAN, Structured Package for Radiation Analysis: Theory and Application". ISAS Report 669, Institute of Space and Aeronautics Science, Sagamihara, Japan, Sep. 1997.

[43] H. Takayanagi, M. Mizuno, K. Fujii, and K. Fujita. "High Enthalpy Flow Diagnostics measured by Laser-Induced Fluorescence to Atomic Nitrogen". June 5 - 122011.

[44] G. Colonna and M. Capitelli. "Self-Consistent Model of Chemical, Vibrational, Electron Kinetics in Nozzle Expansion". Journal of Thermophysics and Heat Transfer, Vol. 15, No. 3, pp. 308-316, July-Sept. 2001.

[45] G. Colonna, , and M. Capitelli. "A Few Level Approach for the Electronic Partition Function od Atomic Systems". Spectrochimica Acta Part B, Vol. 64, pp. 863-873, 2009. 